\title{
@ Price discrimination and product quality under opt-in privacy regulation
}

(i) The corrections made in this section will be reviewed and approved by a journal production editor.

Chiara Conti, Pierfrancesco Reverberi` reverberi@diag.uniroma1.it

Department of Computer, Control and Management Engineering Antonio Ruberti (DIAG), Sapienza University of Rome, Roma, Italy

*Corresponding author at: Via Ariosto, 25 - 00185 Roma, Italy.

\begin{abstract}
We study how an opt-in regime of privacy regulation, which limits the scope for online price discrimination, affects product quality and consumer surplus. When consumers decide to share personal data, they benefit from the complementarity between information and quality, but they pay personalized prices instead of a uniform price. We find that, if the complementarity is strong enough, then product quality is higher with than without the opt-in regime. Privacy regulation may have conflicting effects on consumers with different attitudes towards privacy, and an increase in quality is necessary to improve total consumer surplus. Interestingly, these results hold both under monopoly and imperfect product market competition. We thus recommend that privacy protection measures be grounded in the study of the relation between personal information and product quality.
\end{abstract}

Keywords: Consumer data; Privacy; Opt-in regime; Price discrimination; Product quality

JEL classification: L12; L13; L15; L51

\section{Introduction}

This paper studies how privacy regulation affects product quality and consumer surplus, both under monopoly and imperfect competition. A distinguishing feature of our analysis is that we focus on the interplay between personal information and product quality.

The motivation for the paper is the vibrant academic and policy debate on the need to monitor the massive collection and use of personal data made possible by recent advances in information technology, which have raised privacy concerns. ${ }^{1}$ In the EU, the awareness of privacy risks has led to set out the General Data Protection Regulation (GDPR), which prescribes that the collection and use of personal data be allowed only after explicit consent of data subjects (opt-in regime). ${ }^{2}$ Firms are eager to obtain such data for profiling purposes, so as to set tailored prices, but the opt-in regime makes it easier and less costly for data subjects to preserve anonymity if they intend to do so. Hence, it is more difficult for firms to infer consumers' valuations for the products and price discriminate. ${ }^{3}$ As long as this reduces profits, it may also challenge the incentives to invest in product quality.

In this framework, we shed light on the following issues: Does the opt-in regime, which reduces the room for price discrimination, come at the expense of product quality? Does enhanced privacy protection improve consumer surplus? Ultimately, should consumers be provided with more or less control over personal data? Despite the interest on these issues, economic analyses on the welfare effects of the opt-in regime are scarce (see Section 2 for a review of the relevant literature).

We develop a theoretical model to analyze how the amount of shared data is related to prices, product quality, and consumer surplus. In the baseline model, we consider a monopolist who sells online and invests in quality. We assume that, under the opt-in regime, consumers' willingness to pay (hereafter, WTP) for the product is private information. In this sense, privacy regulation ensures that consumers are anonymous at the outset (at no cost). Having observed the quality level and the uniform price of the product, consumers decide whether or not to share personal data.

We consider two consumer groups. The first consists of consumers who, after evaluating the pros and cons, may opt for sharing data. We refer to these consumers as privacy pragmatists. Instead, the second group includes those who do not leave traces on the web, since they would receive more harm than benefit (e.g. they may have a 'taste for privacy'). ${ }^{4}$ We refer to them as privacy fundamentalists. ${ }^{5}$ Consumers in either group who are anonymous pay the uniform price for the product (if they buy).

When sharing personal data, consumers face a trade-off. On the one hand, they pay personalized prices based on their WTP. We assume that, having obtained personal data, the firm achieves a partial consumer identification (e.g. because it uses a tracking technology with some degree of inaccuracy). Hence, the firm has an imperfect ability to price discriminate and extract consumer surplus.

On the other hand, they enhance their consumption experience. We argue that (perceived) product quality increases with data sharing, and that the benefits of data sharing increase with product quality. Thus, personal information and product quality are complements from the consumers' perspective.

There are several examples of this relationship in online markets. To fix ideas, consider electronic devices or software programs. Generally, firms offer bundles of the core product and add-on services such as delivery terms, after-sale assistance, training and tutoring. Customers are usually provided with add-on services if they share personal data (e.g. by registering on websites). Hence, data sharing increases utility, since it enables consumers to enjoy the benefits of add-on services. ${ }^{6}$ Moreover, these services are more valuable to users when the core product has a higher intrinsic quality. Thus, as long as the 'quality' of an Enterprise Resource Planning (ERP) system is related to a high degree of sophistication or complexity, each user in the organization strongly benefits from training and tutoring services, and the higher the product sophistication the higher the benefit from these services.

In this framework, we find that the opt-in regime may increase or decrease product quality and consumer surplus, depending on the degree of complementarity between information and quality. ${ }^{7}$

Assume, first, that the complementarity is strong enough. In view of the benefits of data sharing, even privacy pragmatists with high WTP may be induced to pay personalized prices. For this purpose, the firm raises both product quality and the uniform price, so that anonymity is less attractive to these consumers. Despite the opt-in regime limits price discrimination, it does not necessarily reduce the incentives to innovate, and may even encourage the firm to provide higher quality.

Privacy pragmatists are the winners, whereas privacy fundamentalists are the losers from a quality improvement under the opt-in regime. Indeed, privacy pragmatists benefit from the higher quality and from data sharing (while avoiding to buy at the uniform price). Instead, privacy fundamentalists, who are not prone to sharing data, are affected by a negative externality. Specifically, the option to choose anonymity for privacy pragmatists puts an upward pressure on the uniform price that reduces the number of privacy fundamentalists who are active in the market. This negative price externality dominates the positive quality externality. Thus, somewhat paradoxically, the opt-in regime hurts exactly those consumers for which protecting personal data is more valuable.

Now, assume that the complementarity between information and quality is weak. Then, privacy pragmatists with high WTP can hardly be induced to share data. Thus, under the opt-in regime, the firm reduces quality (that is costly to obtain). As to privacy pragmatists, those who buy at personalized prices are worse off because of the lower quality, while those who maintain anonymity are often better off. Thus, the net effect of privacy regulation is ambiguous. As to privacy fundamentalists, fewer consumers are active under the opt-in regime, and they pay a higher price for a lower quality. 
We show that an increase in product quality is a necessary condition for the total consumer surplus to increase under the opt-in regime. However, consumer surplus may decrease even in cases with a higher quality. Given that privacy regulation limits price discrimination, the firm may use quality to extract surplus. In this sense, product quality is an effective substitute for personalized prices.

We check the robustness of our results to a number of variants of the baseline model. We show that the main results are not altered when consumer groups are of different sizes, or under an alternative timing where privacy choices are made before the firm sets prices (so that the firm cannot use the uniform price to induce privacy pragmatists to share data). We also show that the opt-in regime more likely improves product quality and consumer surplus in the presence of further privacy protection measures, that is, when the firm has a limited ability to target consumers.

Finally, we extend our model to account for imperfect competition in a vertically differentiated product market. Perhaps surprisingly, we find that the main results of the baseline model still hold. This is mostly because, in a duopoly, the high-quality firm has incentives similar to the monopolist when choosing quality and prices under the opt-in regime, as compared to the benchmark case.

This paper is organized as follows. Section 2 examines the policy debate and reviews the literature. Section 3 introduces the model. Section 4 analyzes the opt-in regime. Section 5 evaluates the effects of this regime on quality and surplus. Section 6 checks the robustness of results. Section 7 deals with product market competition. Section 8 discusses policy implications and outlines future work.

\section{Policy debate and literature review}

Privacy regulation imposes restrictions on the collection and/or use of personal data. Specifically, the opt-in regime shaped by the European GDPR applies stringent rules based on explicit consent of data subjects. ${ }^{8}$ Indeed, 'consent should be given by a clear affirmative act establishing a freely given, specific, informed and unambiguous indication of the data subject's agreement to the processing of personal data'. Thus, consent must be voluntary, must reflect a true choice of the data subject, ${ }^{9}$ and must not be disruptive to the use of the service of interest. Moreover, the data subject must at least be notified about the controller's identity, what kind of data will be processed, and how it will be used. ${ }^{10}$

The EU is deemed to have a stricter and more comprehensive privacy policy than the US, where there are a variety of industry-specific rules, but not yet a federal digital privacy law. Recently, data protection has emerged at the center stage, following breaches of personal data of millions of Internet users. Many have argued that, to restore trust in the digital economy, companies like social networks with global footprint should be regulated as to how they collect and use personal data. ${ }^{11}$

Despite the growing concern about consumer privacy, there is no clear consensus among scholars on the desirability of data protection. Since the pioneering works of Stigler (1980) and Posner (1981), economists have long investigated how consumer privacy affects economic efficiency (for a review, see Acquisti et al., 2016). Recent studies are related to digital technologies and online privacy, and consider different uses of personal data as well as different privacy regimes.

A first literature strand examines the linkage between privacy protection and price discrimination. Taylor and Wagman (2014), among others, assume that under privacy regulation consumers remain anonymous, and firms charge uniform prices. They find that consumer surplus in oligopoly may be higher with no privacy, since tailored pricing increases competition (see also the seminal papers on spatial competition and price discrimination of Thisse and Vives, 1988, and Liu and Serfes, 2004). ${ }^{12}$

Some papers introduce an opt-out choice for consumers, who can protect privacy at a 'hiding cost'. Belleflamme and Vergote (2016) show that, in a monopoly where the firm has an imperfect ability to price discriminate, consumers may be better off when they cannot hide personal data. Montes et al. (2019) study the welfare effects of consumer privacy costs, depending on the industry structure. They also analyze the role of data brokers, and the implications of exclusivity deals to sell consumer data

In other papers (Villas Boas, 2004; Acquisti and Varian, 2005), consumers make repeat purchases. In anticipation of lower future prices, consumers may opt for strategic waiting, which is negative for the firm. On the other hand, Conitzer et al. (2012) show that consumers will choose to anonymize if they can do so for free, which is positive for the firm, since they do not delay purchasing.

Along with this strand, we study how privacy regulation affects tailored pricing. However, in line with the GDPR, we require that consumers give their consent for firms to use personal data (absent regulation, firms know the WTP of those who leave traces of their web activities). In our model, privacy pragmatists may be induced to share data, even if they could preserve anonymity at no cost. Actually, by choosing anonymity, they are losing the benefits of an improved (perceived) quality.

A second strand of literature focuses on big tech platforms (such as Facebook or Google) that make profit by collecting and using consumer data to create value for third parties. ${ }^{13}$ Consumers are usually assumed to choose how much personal data to share. Casadesus-Masanell and Hervas-Drane (2015) study how the provision and disclosure of consumer data shape competitive interaction of firms. Choi et al. (2019) assess the importance of information externalities in data collection from a welfare perspective. $^{14}$

To our knowledge, very few theoretical papers study how privacy regulation affects the incentives to innovate. ${ }^{15}$ Haberer et al. (2020) explore how personal data markets may encourage users to port their data and sell them directly, ${ }^{16}$ and how this impacts on the platform's incentive to invest in service quality. Lefouili and Toh (2019) share the idea that there is a complementarity between information and quality. They assess the effects of a cap on data disclosure to third parties, and find that it improves service quality when it greatly increases the sensitivity of demand to changes in quality.

We depart from these papers in at least two essential ways. First, consumer data are used for price discrimination, rather than targeted advertising. Second, in platform models, personal data is often created as a byproduct of service usage (when consumers patronize the firm, they automatically share personal data). Consistent with the optin regime shaped by the GDPR, in our model some privacy pragmatists may decide not to share data and still purchase the product at the uniform price.

Thus, we consider a combination of endogenous information sharing (under privacy regulation), price discrimination, and quality investment, which has not been addressed in the relevant literature. ${ }^{17}$

\section{The model}

In this section, we present the set-up of the baseline model. There are two types of agents, consumers and the firm, who may interact in the presence or absence of a consent-based privacy regime.

Consumers. Let the gross utility of a consumer of type $\theta$ who buys a product of quality $q$ be:

$U(\theta)=\theta q+\gamma q^{k} x$

where $x$ indicates the binary choice between sharing personal data $(x=1)$ or not $(x=0)$. Thus, the utility from buying depends on two additively separable terms. The first term, $\theta q$, is a stand-alone benefit measuring the consumer's valuation of the intrinsic quality of the product. The second term, $\gamma q^{k} x$, is an information benefit related to the consumer's decision on whether or not to share data. ${ }^{18}$ On the other hand, the utility from not buying is normalized to zero.

When sharing personal data, type $\theta$ enjoys a better purchasing experience. Indeed, the benefit of information provision increases with product quality, and the increase is stronger the higher the values of $\gamma$ and $k$, with $\gamma \in(0,1)$ and $k>0$. Therefore, parameters $\gamma$ and $k$ affect the strength of complementarity between information and quality. ${ }^{19}$

Consumers have unit demand. We assume that types $\theta$ are uniformly distributed between 0 and 1 with unit density in two groups with different attitudes towards privacy. ${ }^{20}$ Given the information benefit, consumers in the first group may decide to share data (i.e. they are privacy pragmatists). Instead, consumers in the second group prefer to keep their WTP private and maintain anonymity, since they receive more harm than benefit from sharing data (i.e. they are privacy fundamentalists). Hence, variable $x$ in (1) is equal to zero for all types in the second group. ${ }^{21}$ 
The firm. A monopolist sets the quality and the prices of the product that it sells online. Let $C(q)=q^{2} / 2$ be the cost to obtain quality $q,{ }^{22}$ while we normalize marginal production costs to zero.

Consumers (in either group) who do not share data and remain anonymous pay the same uniform price $P$, with no information benefit. Instead, a consumer of type $\theta$ who shares data has to pay a personalized price $P(\theta)$ for buying. We assume that the firm can obtain a conservative estimate of the WTP of consumers who share data (e.g. due to an inaccurate tracking technology). Thus, the firm has an imperfect ability to extract consumer surplus, measured by parameter $\alpha$, with $\alpha \in(0,1)$. ${ }^{23}$ This means that, when type $\theta$ shares data, a monopolist with ability $\alpha$ sets $P(\theta)=\alpha\left(\theta q+\gamma q^{k}\right)$.

We assume that, if consumers share data, then the firm can price discriminate at the time of sale. Indeed, after the consumer has given explicit consent, the firm can instantly combine both publicly available and privately collected information (including demographic factors, clickstream data, and purchase history), to profile the consumer before purchase (see e.g. Montgomery et al., 2004). ${ }^{24}$

We also assume that, for online price discrimination to be effective, a consumer who shares data cannot access prices targeted at others, and cannot easily resell products Indeed, online purchases of registered users are a sort of non-transferable contracts with identified beneficiaries. ${ }^{25}$ Although the Internet may have expanded the chances to resell items, there may be high transaction costs in second-hand markets. An example is digitalized, DRM-protected content (e-books, music, and movies) that cannot be resold as easily as when it was held on tangible media (books, CDs, and DVDs). ${ }^{26}$

Timing. The timing of the game proceeds as follows:

Stage 1. The monopolist undertakes R\&D and sets product quality.

Stage 2. The monopolist manufactures the product and sets the uniform price.

Stage 3. Consumers in the first group decide whether to share personal data or not.

Stage 4. The firm sets personalized prices for consumers who have shared personal data.

Stage 5. Consumers in the first group buy at either personalized prices or the uniform price. Consumers in the second group decide whether to buy at the uniform price or not. $\underline{27}$

Consumers in the second group deeide whether to buy at the uniform priee or not. ${ }^{27}$

We solve the game backwards. In Section 4, we find the equilibrium outcome under the opt-in regime. In Section 5, we compare this outcome with the benchmark case without privacy regulation, where the firm knows first-group consumers' WTP. All proofs are in Appendix A (unless otherwise stated).

\section{Opt-in privacy regime}

In our model, privacy regulation endows consumers with anonymity. We find two types of equilibria under the opt-in regime. When the complementarity between information and quality is weak (so that the information benefit is small), there is an interior solution where high-WTP consumers in the first group remain anonymous. When the complementarity is strong, there is a corner solution where the firm chooses product quality and the uniform price to induce all first-group consumers to share data.

Consumers' decisions. Let $\widetilde{\theta}=P / q$ be the marginal consumer in the second group, who is indifferent between buying at the uniform price and not buying. Types $\theta$ such that $\theta \in[\widetilde{\theta}, 1]$ are active. Then, let $\widehat{\theta}$ be the consumer in the first group who is indifferent between sharing data, thereby paying $P(\theta)$, or not, thereby paying $P$. Thus, we have $(1-\alpha)\left(\widehat{\theta} q+\gamma q^{k}\right)=\widehat{\theta} q-P$, and hence we find:

$$
\widehat{\theta}=\frac{P}{\alpha q}+\frac{(1-\alpha)}{\alpha} \gamma q^{(k-1)}
$$

Types $\theta$ such that $\theta \in(\widehat{\theta}, 1]$ remain anonymous, whereas types $\theta$ such that $\theta \in[0, \widehat{\theta}]$ share data (the first group is fully covered, since the firm has zero cost and cannot extract all consumer surplus)

Pricing. At the second stage, for a given quality $q$, and while anticipating consumers' decisions, the firm sets the uniform price $P$ that maximizes:

$$
\Pi(P, q)=\int_{0}^{\widehat{\theta}} P(\theta) d \theta+\int_{\widehat{\theta}}^{1} P d \theta+\int_{\widetilde{\theta}}^{1} P d \theta-C(q)
$$

subject to $\widehat{\theta} \leq 1$ and $\widetilde{\theta} \geq 0$. Since profit is concave in $P$ then, from the FOC, the optimal price is:

$$
P(q)=\frac{\alpha q\left(2+\gamma q^{(k-1)}\right)}{(1+2 \alpha)}
$$

provided that $\hat{\theta}(P(q))<1$. By substituting (4) respectively in $\widehat{\theta}$ and $\widetilde{\theta}$, we obtain:

$$
\widehat{\theta}=\frac{2 \alpha+\gamma q^{(k-1)}\left(1+2 \alpha-2 \alpha^{2}\right)}{\alpha(1+2 \alpha)}
$$

$$
\widetilde{\theta}=\frac{\alpha\left(2+\gamma q^{(k-1)}\right)}{(1+2 \alpha)}
$$

It follows from (5) that $\hat{\theta}(P(q))<1$ as long as $q<\left(\frac{\alpha(2 \alpha-1)}{\gamma\left(1+2 \alpha-2 \alpha^{2}\right)}\right)^{\frac{1}{(k-1)}} \equiv \bar{q}$. On the other hand, when $q \geq \bar{q}$, we may find a corner solution where the optimal price is-: ${ }^{28}$

$P(q)=\alpha q-(1-\alpha) \gamma q^{k}$ 
Quality choice. At the first stage, the firm chooses product quality. To obtain closed-form solutions for quality levels, we have to set a value for parameter $k$, thereby determining the concavity/convexity of the utility function. This, in turn, affects the way in which the firm can 'strategically' use quality. Substituting (4) into (2) and taking the first derivative with respect to (hereafter, WRT) $q$, we obtain:

$$
\partial \hat{\theta} / \partial q=(k-1) \gamma q^{(k-2)}\left(\frac{1+2 \alpha-2 \alpha^{2}}{\alpha(1+2 \alpha)}\right)
$$

It follows that $\partial \hat{\theta} / \partial q>0$ as long as $k>1$, that is, the firm can use quality to induce first-group consumers to share personal data if and only if the utility function is strictly convex in quality.

Consider, for a moment, the case where $k=1$ (i.e. utility is linear in quality). Then, from (9), $\partial \hat{\theta} / \partial q=0$ holds, so that a higher quality does not increase the number of firstgroup consumers who share data. Solving the model, we find that, when $k=1$, product quality is lower (and the uniform price is higher) with than without the opt-in regime. In such a case, privacy regulation should not be enforced, since it reduces consumer surplus and social welfare. ${ }^{30}$

Hereafter, we focus on the case where $\partial \hat{\theta} / \partial q>0$, which occurs when the utility of sharing data increases more than proportionally with quality (i.e. $k>1$ ). In this case, the firm can use product quality to obtain consumer data. This is the most interesting case for our analysis, since we can study whether and when, as a non-trivial response to a privacy regime limiting price discrimination, the firm uses quality to extract consumer surplus. For reasons of tractability, we set $k=2 \cdot{ }^{31}$

Since $k$ is fixed, then the complementarity between information and quality is shaped solely by parameter $\gamma$. We assume that the following conditions hold: (H1) $\alpha \geq 1 / 2$; (H2) $\alpha \gamma<1 / 2$. If $(H 1)$ is not met then the ability to extract consumer surplus is very low, and uniform pricing is more profitable than personalized pricing. This contrasts with the increased demand for personal data to profile consumers (Montes et al., 2019). Condition (H2) limits the combined effect of the ability to price discriminate and the strength of complementarity. For the given cost function, $(H 2)$ ensures the existence of an optimal quality level.

Consider an interior solution. Then, the optimal quality maximizes (3), where the uniform price is given by (4), $\hat{\theta}$ by (5), and $\tilde{\theta}$ by $(6)$, with $k=2$. Let $\Gamma=(1+2 \alpha(1-2 \gamma))$ and $\Lambda=\left(1+2 \alpha\left(1-\alpha^{2}\right)\right)$. Lemma- 1 summarizes the equilibrium outcome (subscript $I$ stands for interior solution).

[Instruction: Lemma 1. (in bold, followed by ".")]Lemma 1 In an interior solution, product quality is $q_{I}=\frac{\alpha\left(\Gamma-\sqrt{\Gamma^{2}-12 \gamma^{2} \Lambda}\right)}{3 \gamma^{2} \Lambda}$ and the uniform price is $P_{I}=\frac{\alpha^{2}\left(\Gamma-\sqrt{\Gamma^{2}-12 \gamma^{2} \Lambda}\right)\left(6 \Lambda \gamma+\alpha\left(\Gamma-\sqrt{\Gamma^{2}-12 \gamma^{2} \Lambda}\right)\right)}{9 \Lambda^{2} \gamma^{3}(1+2 \alpha)}$

Consider now a corner solution. Then, the optimal quality maximizes (3), where the uniform price is given by (7), $\tilde{\theta}$ by (8), and $\hat{\theta}=1$, with $k=2$. Let $Z=\left(2 \gamma\left(4 \alpha-2 \alpha^{2}-1\right)-1\right)$ and $\Psi=6 \gamma^{2}(1-\alpha)^{2}$. Lemma- 2 summarizes the equilibrium outcome (subscript $C$ stands for corner solution).

[Instruction: Lemma 2. (in bold, followed by ".")]Lemma 2 In a corner solution, product quality is $q_{C}=\frac{Z+\sqrt{Z^{2}+\Psi\left(3 \alpha-2 \alpha^{2}\right)}}{\Psi}$ and the uniform price is $P_{C}=\frac{\alpha \Psi\left(\mathrm{Z}+\sqrt{Z^{2}+\Psi\left(3 \alpha-2 \alpha^{2}\right)}\right)-(1-\alpha) \gamma\left(\mathrm{Z}+\sqrt{Z^{2}+\Psi\left(3 \alpha-2 \alpha^{2}\right)}\right)^{2}}{\psi^{2}}$ [Instruction: Equation of Pc in Lemma 2 is misaligned].

Let $\bar{\gamma}(\alpha)=\frac{2\left(-4 \alpha^{3}+6 \alpha^{2}-1\right)}{\left(-12 \alpha^{4}+10 \alpha^{3}+12 \alpha^{2}-12 \alpha+7\right)}$. Proposition 1 shows that, if the complementarity between information and quality is weak (i.e. $\left.\gamma<\bar{\gamma}(\alpha)\right)$ then the firm sets product quality and the uniform price so that, in equilibrium, there is an interior solution where high-WTP consumers in the first group preserve anonymity. Instead, if the complementarity is strong (i.e. $\gamma \geq \bar{\gamma}(\alpha)$ ) then product quality and the uniform price are such that, in equilibrium, there is a corner solution where all first-group consumers share data and pay personalized prices.

[Instruction: Proposition 1. (in bold, followed by ".")]Proposition 1 There is a critical value $\bar{\gamma}(\alpha)$ of $\gamma$ such that, when $\gamma<\bar{\gamma}(\alpha)$, high-WTP consumers in the first group maintain anonymity. Instead, when $\gamma \geq \bar{\gamma}(\alpha)$, all first-group consumers share data.

From Proposition 1 , we have $\frac{\partial \bar{\gamma}(\alpha)}{\partial \alpha}>0$. Thus, if the ability to extract consumer surplus is low $(\alpha \rightarrow 1 / 2)$, then even high-WTP consumers are eager to share data (since $\bar{\gamma}(\alpha) \rightarrow 0)$. As the ability increases $(\alpha \rightarrow 1)$, this outcome becomes less likely $(\bar{\gamma}(\alpha) \rightarrow 0.4)$, because the information benefit has to be large enough to offset the utility loss due to personalized prices.

\section{Comparison of results with and without privacy regulation}

We now assess the effects of the opt-in regime on product quality and on consumer surplus. For this purpose, we first consider the benchmark case without privacy regulation. Then, we compare the equilibrium outcomes under the opt-in regime and in the benchmark case.

\subsection{Benchmark case (no privacy regulation)}

In the absence of privacy regulation, the firm knows the types of consumers in the first group. In their web activities, they release data that the firm can use to target them even without an explicit consent (stage 3 of the game is removed). Instead, consumers in the second group are anonymous, since they do not leave traces on the web. In this framework, the firm charges personalized prices to all first-group consumers, while it acts as a standard uninformed monopolist for the second group.

Let $\tilde{\theta}=P / q$ be the marginal consumer in the second group. At stage 2 , the firm sets the price that maximizes $\int_{\tilde{\theta}}^{1} P d \theta$, that is, $P=q / 2$. Then, we find $\tilde{\theta}=1 / 2$, and the firm serves the top half of the second group. Proposition $\underline{2}$ shows that, for a given quality, the firm sets a uniform price that is not lower under the opt-in regime than in the benchmark case (see Belleflamme and Vergote, 2016). The aim is to make the option of maintaining anonymity less attractive for consumers.

[Instruction: Proposition 2. (in bold, followed by ".")]Proposition 2 For a given quality, the uniform price is not lower with than without the opt-in regime.

At stage 1, the firm sets product quality to maximize profit. Lemma $\underline{3}$ summarizes the equilibrium outcome (subscript $B$ stands for benchmark case).

[Instruction: Lemma 3. (in bold, followed by ".")]Lemma 3 In the benchmark case without privacy regulation, product quality is $q_{B}=\frac{(2 \alpha+1)}{4(1-2 \alpha \gamma)}$ and the uniform price is $P_{B}=\frac{(2 \alpha+1)}{8(1-2 \alpha \gamma)}$.

In what follows, we focus on the case where the opt-in regime is binding. Privacy regulation is not binding if the firm chooses the same quality and prices as in the benchmark case, and nonetheless we find a corner solution where all first-group consumers share personal data. Intuitively, this occurs if the complementarity between information and quality is very strong, so that the information benefit is so large that no consumer in the first group prefers anonymity, independent of the privacy regime.

More formally, given the optimal quality and prices in the benchmark case, privacy regulation is not binding if even the highest-WTP type $(\theta=1)$ has a lower utility by maintaining anonymity and buying at the uniform price under the opt-in regime. For this to occur, condition $(1-\alpha)\left(q_{B}+\gamma q_{B}{ }^{2}\right)>q_{B}-P_{B}$ must hold, which, in turn, is fulfilled when $\gamma>\frac{2(2 \alpha-1)}{\left(6 \alpha^{2}-3 \alpha+1\right)} \equiv \gamma_{U}(\alpha)$. Thus, in what follows, we compare quality and prices with and without privacy regulation in the region where the opt-in regime is binding, that is, when $\gamma \leq \gamma_{U}(\alpha)$. 
Consider first product quality. Proposition $\underline{3}$ shows that, in an interior solution, quality is lower under the opt-in regime than in the benchmark case. Indeed, if the complementarity between information and quality is weak (i.e. $\gamma<\bar{\gamma}(\alpha)$ ) then, for a given quality, first-group consumers would have a small information benefit. Since quality is costly, then the firm does not find it profitable to improve quality to the extent that high-WTP consumers are induced to share data and pay personalized prices. Thus, the firm reacts to the profit loss caused by the opt-in regime, which limits the scope for price discrimination, by reducing quality.

[Instruction: Proposition 3. (in bold, followed by ".")]Proposition 3 Let $\gamma<\bar{\gamma}(\alpha)$, so that at least some high-WTP consumers in the first group do not share personal data. Then, product quality is lower under the opt-in regime than in the benchmark case, that is, $q_{I}<q_{B}$.

When $\gamma \geq \bar{\gamma}(\alpha)$, there is a corner solution where first-group consumers share data. Proposition $\underline{4}$ then shows that product quality may be higher under the opt-in regime than in the benchmark case. This occurs if the complementarity between information and quality is strong enough (i.e. $\gamma \geq \gamma_{L}(\alpha)=\frac{(4 \alpha-2)}{2 \alpha^{2}-\alpha+3}>\bar{\gamma}(\alpha)$ ). Indeed, for a given quality, firstgroup consumers want to share data to gain a large information benefit. Hence, the firm can use product quality as a tool to obtain high-WTP consumer data. Thus, privacy regulation may lead the firm to improve quality to reduce the profit loss relative to the benchmark case. Although privacy regulation reduces profit, it does not necessarily result in lower incentives to invest in quality.

[Instruction: Proposition 4. (in bold, followed by ".")]Proposition 4 Let $\gamma \geq \bar{\gamma}(\alpha)$, so that all first-group consumers share data. Then, product quality is higher under the opt-in regime than in the benchmark case as long as the complementarity between information and quality is strong enough. Formally, $q_{C} \geq q_{B}$ holds if $\gamma \geq \gamma_{L}(\alpha)>\bar{\gamma}(\alpha)$.

Fig. 1 illustrates the results. In the figure, the light gray area highlights the combinations of parameter values such that $\gamma_{L}(\alpha)<\gamma<\gamma_{U}(\alpha)$, for which product quality is higher under the opt-in regime. Conversely, the white area includes parameter combinations for which quality is lower under the opt-in regime. Finally, in the dark gray area the opt-in regime is not binding (the dashed area at the top right corner of Fig. 1 as well as of all other figures is excluded by assumption $H 2$ ).

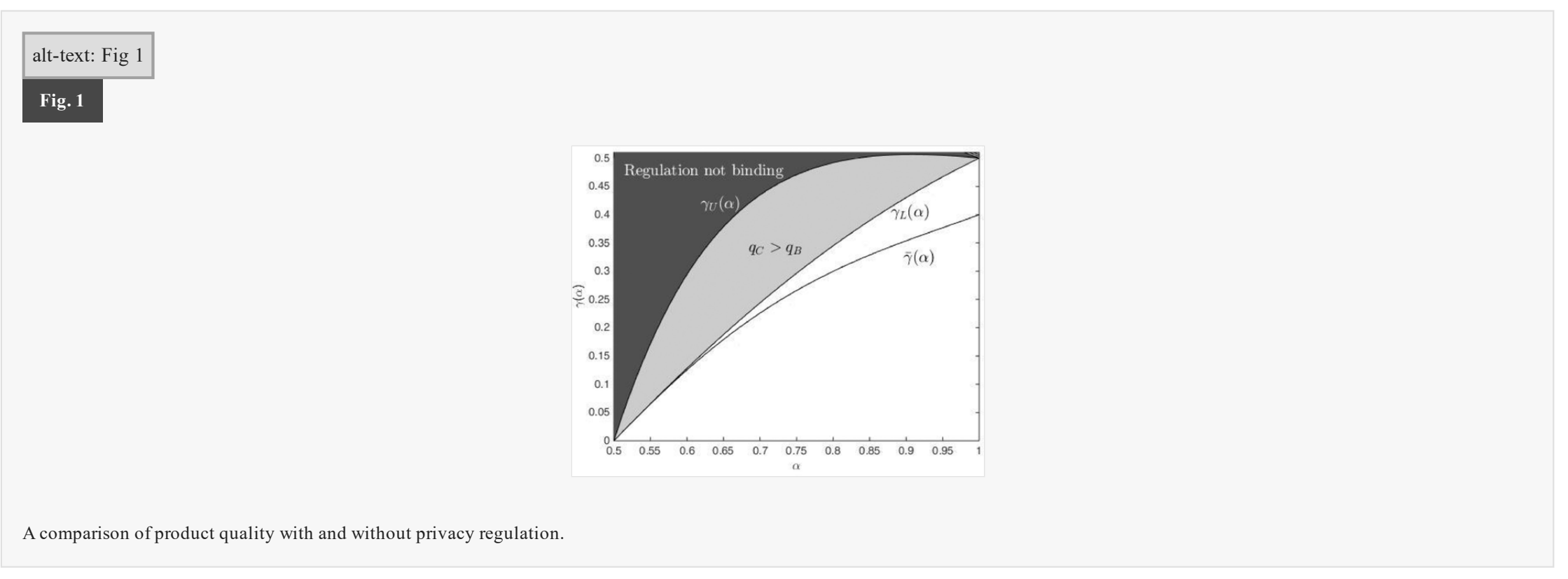

\subsection{Consumer surplus}

As to consumer surplus, we show that the opt-in regime may have conflicting effects on consumers with different attitudes towards privacy, so that there may be winners and losers from the regime. Let $C S_{1}=\int_{0}^{\widehat{\theta}}\left(\theta q+\gamma q^{2}-P(\theta)\right) d \theta+\int_{\widehat{\theta}}^{1}(\theta q-P) d \theta$, with $\widehat{\theta} \leq 1$, be the consumer surplus for group 1,CS $=\int_{\tilde{\theta}}^{1}(\theta q-P) d \theta$ be the surplus for group 2, and $C S=C S_{1}+C S_{2}$ the total consumer surplus. Let $\triangle C S=\Delta C S_{1}+\Delta C S_{2}$ be the change in total consumer surplus with and without privacy regulation.

Consider first group 1 (i.e. privacy pragmatists). We show that, for consumers sharing data (and paying personalized prices), the change in surplus between the opt-in regime and the benchmark case is simply driven by the change in quality. Hence, in a corner solution, consumer surplus for group 1 increases when the opt-in regime improves quality. In an interior solution, some high-WTP consumers in the first group prefer not to share data. Compared to the benchmark case, the change in surplus for these consumers has an ambiguous sign, depending on $\alpha$ and $\gamma$, which can be positive even if product quality is lower. Moreover, this gain in surplus may outweigh the loss in surplus for consumers sharing data. Thus, in an interior solution, consumer surplus for group 1 may increase or decrease relative to the benchmark case.

Consider now group 2 (i.e. privacy fundamentalists). We find that the opt-in regime generates a negative externality on these consumers, who are not prone to sharing data Indeed, the option to choose anonymity for first-group consumers puts an upward pressure on the uniform price that reduces demand and consumer surplus for the second group. Even when group 2-consumers benefit from the quality improvement induced by the opt-in regime, the negative price externality offsets the positive quality externality. Thus, somewhat paradoxically, the opt-in regime hurts exactly those consumers for which protecting personal data is more valuable. Proposition $\underline{5}$ summarizes the results.

[Instruction: Proposition 5. (in bold, followed by ".")]Proposition 5 Compared to the benchmark case, first-group consumers may be better off under the opt-in regime, and they are always better off if privacy regulation improves quality. Conversely, the opt-in regime always reduces demand and consumer surplus for the second group.

Finally, we study the impact of the opt-in regime on total consumer surplus. We find that this impact depends heavily on how the regime affects product quality. If the opt-in regime reduces quality, then consumers in group 1 who share data are worse off. Hence, in a corner solution, there are no winners, since both groups are harmed by the privacy regime. Instead, in an interior solution, consumers in group 1 who do not share data may be better off. Nonetheless, Proposition $\underline{6}$ shows that this positive effect does not offset the negative effect of the opt-in regime on the other consumers.

[Instruction: Proposition 6. (in bold, followed by ".")]Proposition 6 If product quality is lower under the opt-in regime than in the benchmark case, then the total consumer surplus is also lower under privacy regulation.

Corollary $\underline{1}$ follows directly from Proposition $\underline{6}$.

[Instruction: Corollary 1. (in bold, followed by ".")]Corollary 1 An increase in product quality is a necessary condition for the total consumer surplus to increase under the opt-in regime.

Proposition $\underline{5}$ has shown that, if the opt-in regime improves quality (this may only occur in a corner solution), then first-group consumers are better off. Proposition $\underline{7}$ shows that, if the complementarity between information and quality is strong enough (i.e., $\gamma>\gamma^{*}(\alpha)$, where $\gamma^{*}(\alpha)$ is defined in the proof of the proposition), so that the information benefit is large, then the increase in consumer surplus for group 1 offsets the decline in surplus for group 2. Note that, as $\gamma$ rises, the decline in $C S_{2}$ becomes small relative to the increase in $C S_{1}$, since a strong complementarity makes it easier to obtain consumer data, with a lower upward pressure on the uniform price.

[Instruction: Proposition 7. (in bold, followed by ".")]Proposition 7 If the complementarity between information and quality is strong enough, then the increase in quality under the opt-in regime leads to a higher total consumer surplus than in the benchmark case. Formally, if $\gamma$ is such that $\gamma>\gamma^{*}(\alpha)$, then we have that $\Delta C S=\Delta C S_{1}+\Delta C S_{2}>0$.

The light gray (respectively, white) area in Fig. 2 highlights the values of $\alpha$ and $\gamma$ for which the total consumer surplus increases (decreases) under the opt-in regime, while in the dark gray area the regime is not binding. Fig. 2 also shows that an increase in quality (that occurs for $\gamma>\gamma_{L}(\alpha)$ ) is necessary, but not sufficient, to improve total consumer surplus. ${ }^{32}$ 


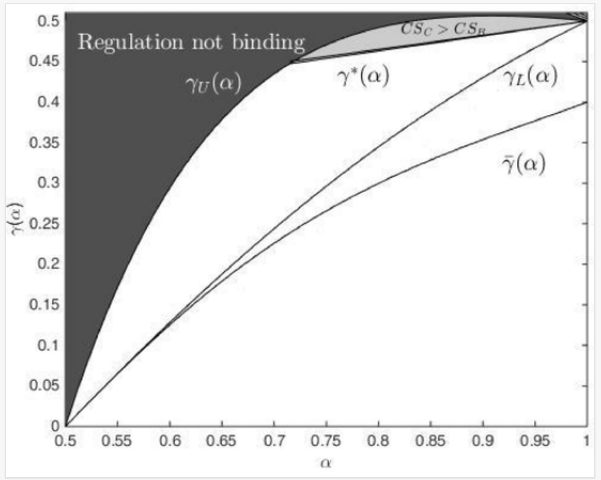

\section{Discussion and extensions}

In this section, we check the robustness of our results to a number of variants of the baseline model. First, we allow for consumer groups of different sizes. Second, we consider an alternative timing of the game where the firm sets prices after observing consumers' privacy choices. Third, we discuss the effects of further privacy protection measures limiting the firm's ability to target consumers.

\subsection{Consumer groups of different sizes}

Changes in the relative sizes of consumer groups may affect the overall impact of the opt-in regime on consumers, because they may alter the firm's incentives to use product quality and the uniform price to induce first-group consumers to share personal data. Nonetheless, we show that our main findings do not depend on the assumption that consumer groups are of the same size, since they still hold when sizes are different (unless group 2 is extremely large, as we clarify in footnote 34).

Let the types in group 1 (respectively, group 2) be uniformly distributed between 0 and 1 with unit mass (mass $n$ ). ${ }^{33}$ Fig. 3 shows the effects of the opt-in regime on product quality and consumer surplus for different values of $n$ (either higher or lower than 1), depending on $\alpha$ and $\gamma$. Note that, in the region below (above) the critical value $\bar{\gamma}_{S}(\alpha)$, there is an interior (a corner) solution.

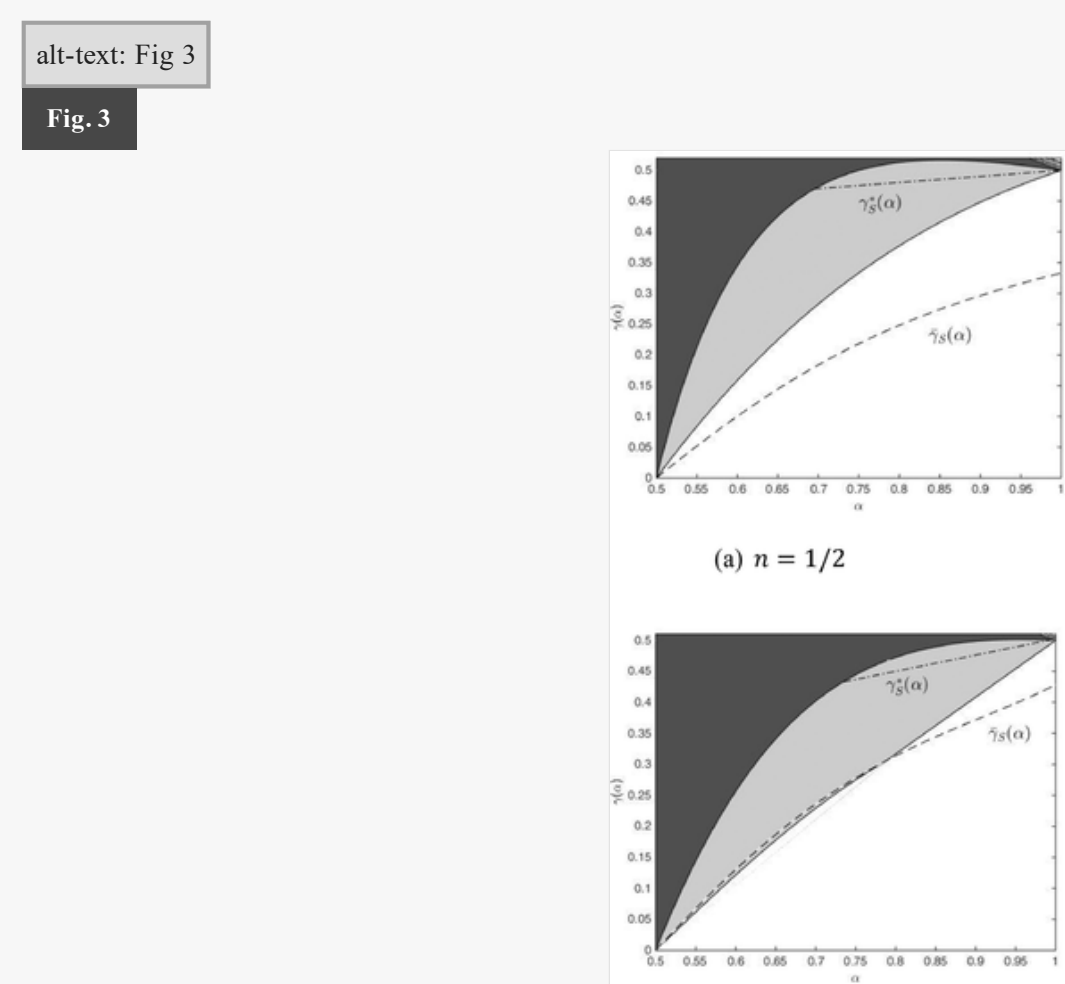

(c) $n=3 / 2$

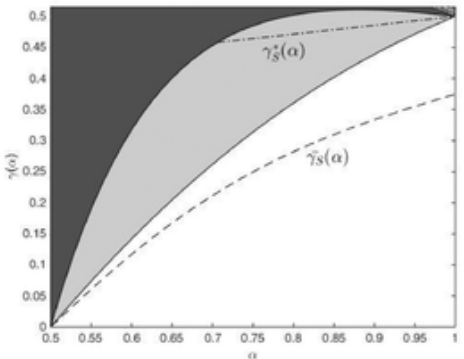

(b) $n=3 / 4$

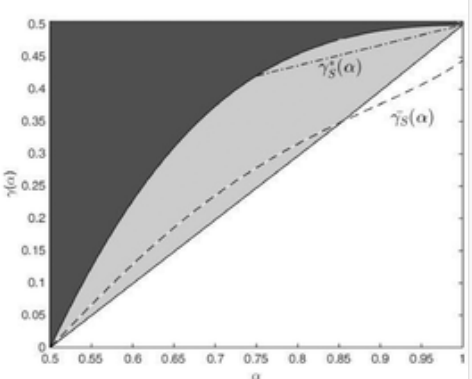

(d) $n=2$

A comparison of product quality and consumer surplus with and without privacy regulation, for different sizes of group 2.

The light gray area indicates the parameter values for which product quality is higher under privacy regulation. Moreover, in this area, the region above the critical value $\gamma_{S}^{*}(\alpha)$ includes all the pairs $(\alpha, \gamma)$ for which privacy regulation also increases consumer surplus. As in the baseline model, an increase in quality is necessary to obtain an increase in consumer surplus.

First, assume that group 2 is smaller than group 1 (see panels (a) and (b) in Fig. 3). Then, compared to the baseline model where groups are of the same size, the firm has higher incentives to increase the uniform price to induce consumers in group 1 to share data, since the revenue loss from group 2 is limited. Therefore, for a given $\alpha$, a corner solution more likely arises due to the increase in price, while higher values of $\gamma$ are needed to find an increase in quality.

Now, assume that group 2 is larger than group 1 (panels (c) and (d) in Fig. 3). Compared to the case where groups are of the same size, while the opt-in regime negatively affects more consumers, the firm has less incentives to increase the uniform price (to preserve revenues from group 2). Hence, an interior solution more likely arises, while lower values of $\gamma$ are needed to find an increase in quality. Different from the baseline model, when $n>1$ the opt-in regime may improve quality even in an interior solution. These facts contribute to explain why, under the opt-in regime, the total consumer surplus may increase even for high values of $n .{ }^{34}$

\subsection{Alternative timing of the game}

Now, assume that consumers make privacy choices before the firm sets prices (i.e. stages 2 and 3 are reversed). Different from the baseline model, the fraction of group 1consumers sharing data is fixed when (at stage 3) the firm sets the uniform price. In turn, privacy choices (at stage 2) are based on product quality and the anticipated price $P^{a}$ (in equilibrium, expectations are fulfilled, and $P^{a}=P$ ).

Assume that the opt-in regime is binding. Solving the game backwards, we find similar results to the baseline model, which are summarized in Proposition $\underline{8}$ (the proofs of this section are in the Online Appendix). Specifically, with a strong complementarity between information and quality (i.e. a large information benefit), all first-group 
[Instruction: Proposition 8. (in bold, followed by ".")]Proposition 8 Assume that consumers make privacy choices before the firm sets prices. Then, there is a critical value $\bar{\gamma}_{T}(\alpha)$ of $\gamma$ such that, when $\gamma<\bar{\gamma}_{T}(\alpha)$, some high-WTP consumers in the first group maintain anonymity. Instead, when $\gamma \geq \bar{\gamma}_{T}(\alpha)$, all first-group consumers share personal data.

Let us now compare the results with and without privacy regulation. As in the baseline model, we find that, when the complementarity between information and quality is sufficiently strong, the opt-in regime improves both product quality and consumer surplus. Moreover, the opt-in regime reduces total consumer surplus as long as it reduces product quality. Proposition $\underline{9}$ summarizes the results.

[Instruction: Proposition 9. (in bold, followed by ".")]Proposition 9 Assume that consumers make privacy choices before the firm sets prices. Then, product quality and consumer surplus are higher under the opt-in regime than in the benchmark case as long as the complementarity between information and quality is strong enough. Moreover, an increase in quality is necessary (but not sufficient) for consumer surplus to increase under the opt-in regime.

Under the alternative timing, there are three main differences relative to the baseline model. Indeed, product quality is always higher with than without privacy regulation in a corner solution, and may also be higher in an interior solution. Moreover, consumer surplus always increases when all first-group consumers share data, and may also increase when some high-WTP types remain anonymous. Finally, even group 2 may benefit from the opt-in regime. This is because the firm has less incentives to raise the uniform price if privacy choices are taken as given when the price is set (see the proof of Proposition $\underline{8}$, and more specifically equation $(O 1)$ ).

Fig. 4 displays the parameter regions where the opt-in regime improves product quality (i.e. the light gray area) and consumer surplus (the light gray area that is above the dashed line). ${ }^{35}$ Compared to the baseline model (see Figs. 1 and 2), the region where product quality (respectively, consumer surplus) increases is smaller (larger). As in the baseline model, a quality improvement under the opt-in regime is necessary, but not sufficient, for an increase in consumer surplus.

\section{alt-text: Fig 4 \\ Fig. 4}

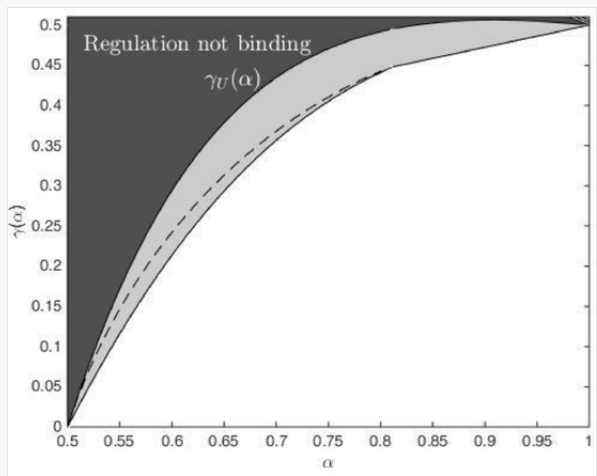

A comparison of product quality and consumer surplus with and without privacy regulation, under the alternative timing.

We also show that the lower ability to affect privacy choices via the uniform price leads to a lower profit relative to the original timing. For this purpose, we evaluate the difference in equilibrium profits in the two model variants, depending on parameter values. Fig. 5 shows a representative case, where the curve $\Delta \Pi=\Pi_{I}-\Pi_{I}^{T}=0$ maps the pairs $(\alpha, \gamma)$ for which, given that there is an interior solution in both variants, profits are the same. We find that $\Delta \Pi>0$ holds in the whole region below the curve, including the feasible region for interior solutions, where $\gamma<\bar{\gamma}(\alpha)$. We find the same qualitative results whatever the combination of (interior or corner) solutions in both variants.

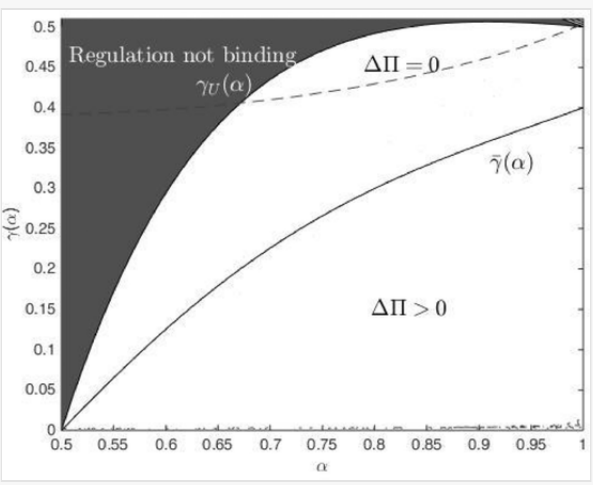

A comparison of profits under the original and the alternative timing, with an interior solution in both variants, i.e. for $\gamma<\bar{\gamma}(\alpha)$

Thus, the firm aims at setting the price in advance, by anticipating privacy choices, while it is more likely that consumer surplus increases under the alternative timing Indeed, in online markets, we often observe pricing offers depending on whether or not consumers share data (with price rebates under data sharing). Generally, consumers are provided with these offers before their privacy choices.

\subsection{Privacy protection and the opt-in regime}

The legal and regulatory framework where the firm operates affects the extent to which it is able to target consumers (think of restrictions on data merge from multiple sources and on data trading, or outright bans on the collection and use of sensitive data). For any given degree of accuracy of the tracking technology, these measures reduce the firm's ability to extract consumer surplus.

In what follows, we analyze how the effects of the opt-in regime are related to the environment in which it is introduced. For this purpose, we study how the sign and the magnitude of the changes in product quality and consumer surplus due to the opt-in regime (when it is binding) depend on $\alpha$.

We draw from Fig. 1 that, for any given $\gamma$, the opt-in regime improves quality for low values of $\alpha$. Thus, when the regulatory framework limits the ability to target consumers, the firm uses quality as a substitute for price discrimination. Note that $\gamma_{L}(\alpha)$, the lowest degree of complementarity such that $q_{C}>q_{B}$, increases in $\alpha .{ }^{36}$ Hence, if the ability to extract consumer surplus is limited, then the firm may find it profitable to improve quality even if the complementarity between information and quality is relatively weak

Furthermore, we can show that, when product quality is lower under the opt-in regime than in the benchmark case, the lower the value of $\alpha$, the smaller the quality gap.

Fig. 2 shows that, when $\gamma$ is high enough, total consumer surplus increases for low values of $\alpha$. In such a case, quality is likely to increase under the opt-in regime (Fig. 1), which, in turn, makes first-group consumers better off. Moreover, when it is less easy to profile consumers (i.e. for a lower $\alpha$ ), the negative effect of the opt-in regime on second-group consumers is smaller. Indeed, first-group consumers are encouraged to share data, thereby reducing the upward pressure on the uniform price. 
This analysis points out that, as long as achieving price discrimination based on consumer profiling is more difficult, introducing the opt-in regime more likely improves product quality, and has a milder impact on consumers harmed by the increase in the uniform price. Therefore, to achieve welfare benefits, the opt-in regime should be enforced jointly with further policy measures aimed at strengthening consumer privacy protection.

\section{Product market competition}

In this section, we analyze how product market competition affects information sharing as well as the strategic use of product quality and the uniform price under privacy regulation. Perhaps surprisingly, we find that the main results of the baseline model still hold under imperfect competition, mostly because the high-quality firm in a duopoly and the monopolist have similar incentives.

Consider two firms, $H$ and $L$, which sell different qualities (to relax price competition), $q_{H}$ and $q_{L}$, with $q_{H}>q_{L}$. Let $C\left(q_{i}\right)=q_{i}^{2} / 2$ be the cost of quality $q_{i}(i=L, H)$, while marginal costs are normalized to zero. Let the gross utility of a type $\theta$ who buys a product of quality $q_{i}(i=L, H)$ be:

$U(\theta)=\theta q_{i}+x \gamma q_{i}^{2}$

where $x \in\{0,1\}$. When purchasing from firm $i(i=L, H)$, type $\theta$ enjoys an additional (information) benefit by sharing personal data $(x=1)$. Consumers have unit demand

Under the opt-in regime, consumers' WTP is private information. There are two consumer groups with different attitudes towards privacy. We assume that types $\theta$ are uniformly distributed between 0 and 1 with unit density in both groups. Consumers in group 1 (privacy pragmatists) may decide to share data, whereas those in group 2 (privacy fundamentalists) prefer to maintain anonymity. Due to an inaccurate tracking technology, firms can obtain a conservative estimate of the WTP of consumers who share data, which is equal to $\alpha\left(\theta q_{i}+\gamma q_{i}^{2}\right)$, with $\alpha<1 .{ }^{37}$. Thus, firms may extract at most a fraction $\alpha$ of the gross utility of types who share data.

After observing qualities and (uniform) prices, consumers in group 1 decide whether to share data (and enjoy the information benefit) and pay personalized prices. The timing of the game is as follows.

Stage 1. Firms set quality levels for their products.

Stage 2. Firms set (non-negative) uniform prices $P_{H}, P_{L}$.

Stage 3. Consumers in group 1 decide whether to share personal data.

Stage 4. Firms offer (non-negative) personalized prices $P_{H}(\theta), P_{L}(\theta)$ to types who share data.

Stage 5. Consumers make purchase decisions.

We solve the game backwards (we relegate the detailed solution to Appendix B). We find that, in equilibrium, all consumers in group 1 buy the high-quality product. Specifically, high-WTP consumers (i.e. all types in $\left[\widehat{\theta}_{1}, 1\right]$, where $\widehat{\theta}_{1}$ is the type who is indifferent between sharing data or not) do not share data and buy high quality at the uniform price, while low-WTP consumers (i.e. types in $\left[0, \hat{\theta}_{1}\right]$ ) always share personal data with both firms and buy high quality at personalized prices.

As to group 2, firm $H$ serves high-WTP consumers (i.e. all types in $\left[\widehat{\theta}_{2}, 1\right]$, where $\widehat{\theta}_{2}$ is the type who is indifferent between buying high or low quality), while firm $L$ only serves low-WTP consumers (i.e. types in $\left[\widehat{\theta}_{L}, \widehat{\theta}_{2}\right]$, where $\widehat{\theta}_{L}$ is the type who is indifferent between buying or not buying at all).

Due to the complexity of the model, we solve for optimal qualities by relying on numerical solutions, for given values of $\alpha$ and $\gamma$ (we check that the SOCs and the relevant constraints hold, and that the opt-in regime is binding). Then, we compute equilibrium prices, profits, and consumer surplus for each group. Tables 1 and 2 respectively show the equilibrium outcomes for some pairs $(\alpha, \gamma)$ consistent with an interior solution (where some high-WTP types in group 1 maintain anonymity) and with a corner solution (where all types in group 1 share data), together with the related outcomes for the benchmark case (where we assume that, absent regulation, both firms know all types in group 1). As in the case of monopoly, interior (corner) solutions do exist for low (high) values of $\gamma$.

\section{alt-text: Table 1}

Table 1

(i) The table layout displayed in this section is not how it will appear in the final version. The representation below is solely purposed for providing corrections to the table. To preview the actual presentation of the table, please view the Proof.

\section{Previous version}

Duopoly - Interior solutions.

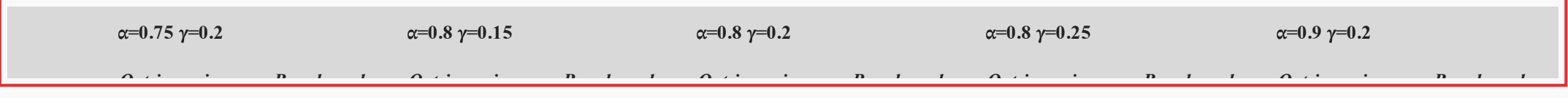

\section{Updated version}

Duopoly - Interior solutions.

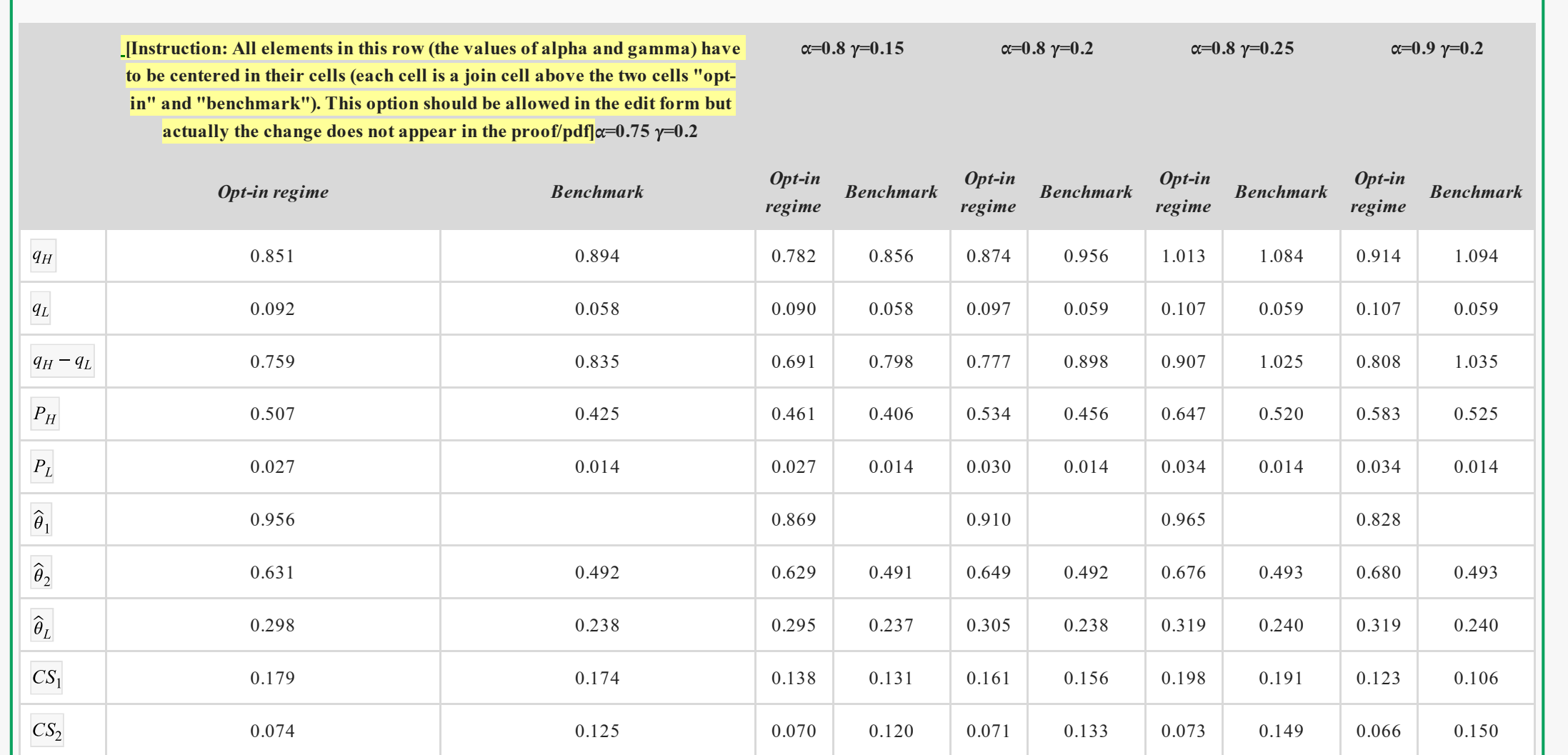




\begin{tabular}{|l|l|l|l|l|l|l|l|l|l|l|l|}
\hline$C S$ & 0.253 & & 0.299 & 0.208 & 0.251 & 0.232 & 0.289 & 0.271 & 0.340 & 0.189 & 0.256 \\
\hline$\Pi_{H}$ & 0.210 & & 0.249 & 0.198 & 0.247 & 0.221 & 0.279 & 0.253 & 0.320 & 0.241 & 0.348 \\
\hline$\Pi_{L}$ & 0.005 & & 0.002 & 0.005 & 0.002 & 0.006 & 0.002 & 0.006 & 0.002 & 0.007 & 0.002 \\
\hline$\Delta q_{H}$ & & -0.043 & & -0.074 & -0.082 & -0.070 & -0.180 \\
\hline$\Delta q_{L}$ & & 0.034 & & 0.032 & 0.039 & 0.048 & 0.048 \\
\hline$\Delta C S$ & & -0.045 & & -0.043 & -0.057 & -0.069 & -0.067 \\
\hline
\end{tabular}

Note: $\Delta q_{H}=q_{H}$ Opt in $-q_{H \text { Benchmark }} ; \Delta q_{L}=q_{L}$ Opt in $-q_{L \text { Benchmark }} ; \Delta C S=C S_{\text {Opt in }}-C S_{\text {Benchmark }}$.

(i) The table layout displayed in this section is not how it will appear in the final version. The representation below is solely purposed for providing corrections to the table. To preview the actual presentation of the table, please view the Proof.

\begin{tabular}{|c|c|c|c|c|c|}
\hline Previous version & & & & & \\
\hline \multicolumn{6}{|l|}{ Duopoly - Corner solutions. } \\
\hline$\alpha=0.75 \gamma=0.4$ & $\alpha=0.8 \gamma=0.3$ & $\alpha=0.8 \gamma=0.4$ & $\alpha=0.8 \gamma=0.48$ & $\alpha=0.9 \gamma=0.4$ & $\alpha=0.9 \gamma=0.5$ \\
\hline
\end{tabular}

\begin{tabular}{|c|c|c|c|c|c|c|c|c|c|c|c|c|}
\hline \multicolumn{13}{|c|}{ Duopoly - Corner solutions. } \\
\hline & \multicolumn{2}{|c|}{$\begin{array}{l}\text { [Instruction: All elements in this row (the values of } \\
\text { alpha and gamma) have to be centered in their cells } \\
\text { (each cell is a join cell above the two cells "opt-in" } \\
\text { and "benchmark"). This option should be allowed in } \\
\text { the edit form but actually the change does not } \\
\text { appear in the proof/pdf] } \alpha=0.75 \gamma=0.4 \\
\text { [Instruction: Elements in this row } \\
\text { (cells with "Opt-in regime" and } \\
\text { "Benchmark") should be } \\
\text { centered]Opt-in regime }\end{array}$} & $\begin{array}{c}\text { Opt-in } \\
\text { regime }\end{array}$ & Benchmark & $\begin{array}{c}\text { Opt-in } \\
\text { regime }\end{array}$ & Benchmark & $\begin{array}{c}\text { Opt-in } \\
\text { regime }\end{array}$ & Benchmark & $\begin{array}{c}\text { Opt-in } \\
\text { regime }\end{array}$ & Benchmark & $\begin{array}{c}\text { Opt-in } \\
\text { regime }\end{array}$ & Benchmark \\
\hline$q_{H}$ & 1.598 & 1.563 & 1.208 & 1.25 & 1.848 & 1.806 & 2.835 & 2.802 & 2.356 & 2.500 & 7.221 & 7.000 \\
\hline$q_{L}$ & 0.074 & $0.06 \underline{0}$ & 1.103 & 0.059 & 0.090 & $0.06 \underline{0}$ & 0.063 & 0.061 & 0.136 & 0.061 & 0.069 & 0.062 \\
\hline$q_{H}-q_{L}$ & 1.524 & 1.503 & 0.105 & 1.191 & 1.758 & 1.746 & 2.772 & 2.741 & 2.220 & 2.439 & 7.152 & 6.938 \\
\hline$P_{H}$ & 0.886 & 0.759 & 0.794 & 0.603 & 1.131 & 0.880 & 1.445 & 1.378 & 1.770 & 1.227 & 3.827 & 3.477 \\
\hline$P_{L}$ & 0.021 & 0.015 & 0.034 & 0.014 & 0.028 & 0.015 & 0.016 & 0.015 & 0.051 & 0.015 & 0.018 & 0.015 \\
\hline$\widehat{\theta}_{1}$ & 1.000 & & 1.000 & & 1.000 & & 1.000 & & 1.000 & & 1.000 & \\
\hline$\hat{\theta}_{2}$ & 0.568 & 0.495 & 0.688 & 0.494 & 0.627 & 0.496 & 0.515 & 0.498 & 0.774 & 0.497 & 0.533 & 0.499 \\
\hline$\widehat{\theta}_{L}$ & 0.277 & 0.243 & 0.329 & 0.241 & 0.306 & 0.244 & 0.255 & 0.246 & 0.376 & 0.245 & 0.265 & 0.248 \\
\hline$C S_{1}$ & 0.485 & 0.463 & 0.252 & 0.243 & 0.497 & 0.467 & 1.082 & $1.05 \underline{0}$ & 0.408 & 0.404 & 3.002 & $2.83 \underline{0}$ \\
\hline$C S_{2}$ & 0.161 & 0.209 & 0.077 & $0.17 \underline{0}$ & 0.143 & 0.239 & 0.343 & 0.364 & 0.083 & 0.326 & 0.799 & 0.888 \\
\hline$C S$ & 0.646 & 0.672 & 0.329 & 0.413 & 0.640 & 0.706 & 1.425 & 1.424 & 0.491 & $0.73 \underline{0}$ & 3.801 & 3.718 \\
\hline$\Pi_{H}$ & 0.442 & 0.457 & 0.308 & 0.374 & 0.507 & 0.554 & 0.875 & 0.887 & 0.615 & 0.838 & 2.398 & 2.413 \\
\hline$\Pi_{L}$ & 0.003 & 0.002 & 0.007 & 0.002 & 0.005 & 0.002 & 0.002 & 0.002 & 0.011 & 0.002 & 0.002 & 0.002 \\
\hline$\Delta q_{H}$ & \multicolumn{2}{|l|}{0.035} & \multicolumn{2}{|c|}{-0.043} & \multicolumn{2}{|c|}{0.043} & \multicolumn{2}{|r|}{0.033} & \multicolumn{2}{|c|}{-0.144} & \multicolumn{2}{|c|}{0.221} \\
\hline$\Delta q_{L}$ & \multicolumn{2}{|l|}{0.014} & \multicolumn{2}{|c|}{1.044} & \multicolumn{2}{|r|}{0.03} & \multicolumn{2}{|r|}{0.002} & \multicolumn{2}{|c|}{0.075} & \multicolumn{2}{|c|}{0.007} \\
\hline$\Delta C S$ & \multicolumn{2}{|l|}{-0.026} & \multicolumn{2}{|c|}{-0.084} & \multicolumn{2}{|c|}{-0.066} & \multicolumn{2}{|r|}{0.002} & \multicolumn{2}{|c|}{-0.239} & \multicolumn{2}{|c|}{0.083} \\
\hline
\end{tabular}

Note: $\Delta q_{H}=q_{H}$ Opt in $-q_{H \text { Benchmark }} ; \Delta q_{L}=q_{L}$ Opt in $-q_{L \text { Benchmark }} ;$ [nstruction: Put here the same equation as in table 1

Imathrm Delta CS $=$ CS_\{Opthspace $\{0.28 \mathrm{em}\}$ in $\}$-CS_\{Benchmark $\}$

Note to table 2 has to be identical to the note to table 1]

We find that the results are qualitatively similar to the baseline monopoly model. When the complementarity between information and quality is weak, consumers' incentive to share personal data is small. Thus, firm $H$ cannot use quality to induce all types in group 1 to share data. Table 1 shows that, in response to the profit loss caused by the opt-in regime, firm $H$ reduces quality relative to the benchmark case. On the other hand, to discourage group 1-consumers from preserving anonymity, firm $H$ raises the uniform price.

Since less consumers in group 2 buy high quality, and more consumers stay out of the market, then consumer surplus for group 2 declines under the opt-in regime. Instead, consumers in group 1 are better off because of lower personalized prices (due to a smaller quality gap than in the benchmark case, which strengthens competition). Overall, this is not enough to outweigh the decline in surplus for group 2, and thus, in an interior solution, total consumer surplus is lower under the opt-in regime.

Instead, when the complementarity is strong, consumers have incentives to share data (due to the large information benefit). Thus, firm $H$ may use product quality (in addition to the uniform price) to induce all group 1-consumers to share data. Table 2 shows that, in response to the profit loss caused by the opt-in regime, firm $H$ raises the uniform price and may increase quality to extract part of the larger surplus for group 1.

As in an interior solution, the opt-in regime makes group 1 better off and group 2 worse off (due to the increase in price). However, in a corner solution, if $\gamma$ is sufficiently high then the positive effect on group 1 may prevail over the negative effect on group 2, leading to a higher total consumer surplus.

Numerical simulations show that an increase in total consumer surplus under the opt-in regime may occur only if there is also an increase in product quality relative to the benchmark case. This is similar to the baseline monopoly model (see Corollary 1). Note that, the higher $\alpha$, the higher must be $\gamma$ to have an increase in total consumer surplus following an increase in $q_{H} .{ }^{38}$ 


\section{Conclusions and policy implications}

We have studied how privacy regulation limiting the scope for online price discrimination affects product quality and consumer surplus, both under monopoly and imperfect competition. We have considered an opt-in regime where consumers decide whether to share personal data or not. If consumers share data, then they gain an additional benefit from buying due to the complementarity between information and quality, but they pay personalized prices instead of a uniform price.

We have shown that, if the complementarity is strong enough, then product quality is higher with than without the opt-in regime. Moreover, an increase in quality is necessary, but not sufficient, to improve total consumer surplus.

Perhaps surprisingly, the main qualitative results hold both under monopoly and under duopoly in the product market. This is mostly because, under the opt-in regime, the high-quality firm in a duopoly has incentives similar to the monopolist (relative to the benchmark case). Indeed, in response to the opt-in regime, product quality and the uniform price may be used as effective means to extract surplus from consumers. The main results are also robust to some variants of the monopoly model, including different sizes for consumer groups, and an alternative timing where consumers make privacy choices before the firm sets prices.

Policy and managerial implications. This study contributes to the worldwide debate on the need for protecting privacy, in the light of technological advances allowing firms to collect and use consumer data for personalized pricing. Our analysis is relevant to policy makers, given that the EU has recently adopted the GDPR, which provides data subjects with the option of managing access to personal data.

We have shown that a consent-based privacy regime may have contrasting effects on consumers, depending on their attitude towards privacy. Interestingly, consumers with a strong taste for privacy are the losers, because of the negative externality of those who may be induced to share personal data. Even when the opt-in regime induces a quality improvement, privacy fundamentalists are harmed by the increase in price. Overall, the impact of the opt-in regime on total consumer welfare is ambiguous.

We argue that the benefits consumers receive from sharing data depend on the strength of complementarity between information and quality. Thus, we recommend that privacy policies be grounded in empirical assessments of the degree of such complementarity, which may be product or industry specific. If the complementarity is strong, then we expect that the opt-in regime has positive welfare effects. Conversely, if the complementarity is weak then the opt-in regime should not be enforced, since it reduces product quality, consumer surplus, and thereby social welfare.

We also recommend that, to expand the welfare benefits of the opt-in regime, a number of ancillary measures be enforced to limit firms' ability to profile consumers from shared data. These measures include, among others, restrictions on data merge from multiple sources and on trading of data, or outright bans on the collection and use of certain types of sensitive data.

In a broader sense, the results obtained shed light on the impact of price discrimination on the incentives to invest in quality and on consumer welfare, which is a central question for R\&D-intensive industries. We have shown that, despite the opt-in regime limits the scope for price discrimination (and thereby may reduce profit), it does not necessarily reduce the incentives to innovate.

Our analysis contributes to explain why firms selling online widely use targeted offers, where the price to be paid depends on whether or not consumers share data. Generally, these offers include a high uniform price in case of anonymity, and a discount price in case of data sharing. Having the full ability to affect privacy choices by a suitable combination of product quality and the uniform price is an ideal situation for managers of such firms, whose business model differs from big tech platforms, for which personal data is created as a byproduct of service usage.

We recommend antitrust authorities to carefully monitor this kind of offers. While it is dubious whether the reward ultimately benefits consumers sharing data, it is likely that the high uniform price harms consumers with a taste for privacy. Thus, agencies should prevent dominant online firms from excessive pricing to induce consumers to share data, by imposing de facto a cap on the price level.

Limitations and future work. We have considered two consumer groups with very different attitudes towards privacy, so that sharing data is not a choice for the group having a taste for privacy. With the advent of the GDPR, consumers may become more aware of the value of personal data, and may be more protected against misuses of such data. Hence, they may be less reluctant to valuing the option of sharing data. With a reduced heterogeneity in preferences for privacy, the opt-in regime may have different effects on quality and consumer surplus, and policy implications may vary accordingly.

A further important point to explore is the endogeneity of the profiling technology. In our model, duopoly firms have the same ability to extract consumer surplus. If firms can invest in improving the accuracy of the tracking technology, or in purchasing consumer analytics from data brokers, then they may choose to have different abilities to profile consumers in equilibrium. While this asymmetry may relax price competition, the impact on product quality is less clear. It is also not clear a priori how a consentbased privacy regime can affect the choice of the profiling technology.

We have assumed that consumers decide whether to share personal data or not when product quality is sunk. Alternatively, they could exercise their option before firms invest in R\&D. In this framework, collecting information on consumers' preferences and valuations is essential for firms to obtain an improved product. In future work, we intend to investigate whether our results on the welfare effects of privacy regulation are robust to this alternative model specification.

Recent technological developments and new business models (both of established firms such as telecom incumbents and of innovative startups) may enable data subjects to commercially benefit from trading their information assets under the opt-in regime. Thus, in an extension of our analysis, we could study whether firms would use the financial incentive as a substitute or complement to product quality as a means to induce consumers to share personal data.

\section{Author statement}

Chiara Conti and Pierfrancesco Reverberi are equally responsible for conceptualization, methodology, formal analysis, writing, and supervision of the research

\section{Acknowledgments}

We wish to thank Marc Bourreau and two anonymous reviewers for very helpful comments and suggestions on an earlier version of the manuscript. We also thank Nicola Meccheri and seminar participants at the 2019 SIEPI Workshop in Rome. Finally, we gratefully acknowledge funding support from Sapienza University of Rome_(grant numbers RP11916B7A9EF5DB, RP120172B96CC6F4)[Instruction: Appendix A and Appendix B have to be inserted (after Acknowledgments and before Supplementary materials). They can be found in the attached file. Appendix A and Appendix B were part of the submitted revised version, but they do not appear in the proof. The authors would like to see how they are expected to appear in the article before publication.].

\section{Supplementary materials}

Supplementary material associated with this article can be found, in the online version, at doi:10.1016/j.infoecopol.2020.100912.

\section{References}

(i) The corrections made in this section will be reviewed and approved by a journal production editor. The newly added/removed references and its citations will be reordered and rearranged by the production team.

Acquisti, A., Taylor, C.R., Wagman, L., 2016. The economics of privacy. J. Econ. Lit. 54, 442-492.

Acquisti, A., Varian, H.R., 2005. Conditioning prices on purchase history. Mark. Sci. 24, 367-381. 
Avenali, A., Matteucci, G., Reverberi, P., 2014. Broadband investment and welfare under functional and ownership separation. Inf. Econ. Policy $28,70-82$.

Belleflamme, P., Vergote, W., 2016. Monopoly price discrimination and privacy: the hidden cost of hiding. Econo. Lett. 149, 141-144.

Bo, P., Manduchi, A., 2017. Disclosure-based price discrimination by information exchange platforms. Inf. Econ. Policy 41, 54-66.

Bourreau, M., de Streel, A., 2018. 2018, The Regulation of Personalised Pricing in the Digital Era. OECD, DAF/COMP/WD, p. 150.

Brandimarte, L., Acquisti, A., Loewenstein, G., 2013. Misplaced confidences: privacy and the control paradox. Soc. Psychol. Personal. Sci. 4, 340-347.

Buehler, S., Gartner, D., Halbheer, D., 2006. Deregulating network industries: dealing with price-quality tradeoffs. J. Regul. Econ. 30, 99-115.

Buehler, S., Schmutzler, A., Benz, M., 2004. Infrastructure quality in deregulated industries: is there an underinvestment problem? Int. J. Ind Organiz. $22,253-267$.

Casadesus-Masanell, R., Hervas-Drane, A., 2015. Competing with privacy. Manage. Sci. 61, 229-246.

Choi, J.P., Jeon, D.S., Kim, B.C., 2019. Privacy and personal data collection with information externalities. J. Public Econ. 173, 113-124.

Conitzer, V., Taylor, C.R., Wagman, L., 2012. Hide and seek: costly consumer privacy in a market with repeated purchases. Mark. Sci. 31, $277-292$.

Crémer, J., Rey, P., Tirole, J., 2000. Connectivity in the commercial Internet. J. Ind. Econ. 433-472 XLVIII.

Dosis, A., Sand-Zantman, W., 2020, The ownership of data, Available at: https://ssrn.com/abstract=3420680.

D’Annunzio, A., Reverberi, P., 2016. Co-investment in ultra-fast broadband access network: Is there a role for content providers? Telecommunications Policy 40, 353-367.

Esteban, L., Hernández, J.M., 2017. Direct advertising and opt-in provision: policy and market implications. Inf. Econ. Policy 39, 15-25.

Ezrachi, A., Stucke, M., 2016. Virtual competition. J. Eur. Compet. Law Practice 7, 585-586.

Foros, O., 2004. Strategic investments with spillovers, vertical integration and foreclosure in the broadband access market. Int. J. Ind Organiz. $22,1-24$.

Ghose, A., Li, B., Liu, S., 2020, Nudging mobile customers with real-time social dynamics, Available at: https://ssrn.com/abstract=3587715.

Goldfarb, A., Tucker, C., 2012. Privacy and innovation. In: Lerner, J., Stern, S (Eds.), Innovation Policy and the Economy. NBER vol 12.

Haberer, B., Krämer, J., Schnurr, D., 2020, Standing on the shoulders of web giants: the economic effects of personal data markets, Available at: $\underline{\text { https://ssrn.com/ab }}$ $\underline{\text { stract }=3141946}$

Katz, M., Shapiro, C., 1985. Network externalities, competition, and compatibility. Am. Econ. Rev. 75, 424-440.

Lefouili, Y., Toh, Y.L., 2019, Privacy regulation and quality investment, https://ideas.repec.org/p/tse/wpaper/31623.html.

Lin, T., 2020, Valuing intrinsic and instrumental preferences for privacy, Available at: $\underline{\text { https: } / / \mathrm{ssrn} . c o m / a b s t r a c t=3406412}$.

Liu, Q., Serfes, K., 2004. Quality of information and oligopolistic price discrimination. J. Econ. Manag. Strategy 13, 671-702.

Liu, Q., Shuai, J., 2016. Price discrimination with varying qualities of information. B.E. J. Econ. Anal. Policy 16, 1093-1121.

Matteucci, G., Reverberi, P., 2017. Drug innovation, price controls, and parallel trade. Int. J. Health Econ. Manage. 17, 159-179.

Matteucci, G., Reverberi, P., 2018. Uniform pricing and product innovation. B.E. J. Theor. Econ. 18 art. n. 20160110.

Montes, R., Sand-Zantman, W., Valletti, T., 2019. The value of personal information in online markets with endogenous privacy. Manage. Sci. 65, $1342-1362$.

Montgomery, A.L., Li, S., Srinivasan, K., Liechty, J.C., 2004. Modeling online browsing and path analysis using clickstream data. Mark. Sci. 23, 579-596.

Nguyen, X., 2014. Monopolistic third-degree price discrimination under vertical product differentiation. Econ. Lett. 125, 153-155.

Posner, R.A., 1981. The economics of privacy. Am. Econ. Rev. 71, 405-409.

Shaw, I., Vulkan, N., 2012, Competitive personalized pricing: an experimental investigation, http://citeseerx.ist.psu.edu/viewdoc/download?doi=10.1.1.490.6911\&r $\underline{\text { ep}}=$ rep $1 \&$ type $=$ pdf.

Stigler, G., 1980. An introduction to privacy in economics and politics. J. Legal Stud. 9, 623-644.

Taylor, C.R., Wagman, L., 2014. Consumer privacy in oligopolistic markets: winners, losers and welfare. Int. J. Ind Organiz. 34, 80-84.

Thisse, J.F., Vives, X., 1988. On the strategic choice of spatial price policy. Am. Econ. Rev. 78, 122-137.

Tucker, C.E., 2014. Social networks, personalized advertising, and privacy controls. J. Mark. Res. 51, 546-562.

Ulph, D., Vulkan, N., 2007, Electronic commerce, price discrimination and mass customisation, http://citeseerx.ist.psu.edu/viewdoc/download?doi=10.1.1.541.381 2\&rep=rep1\&type $=$ pdf.

Villas-Boas, J.M., 2004. Price cycles in markets with customer recognition. RAND J. Econ. 35, 486-501.

Wathieu, L., Friedman, A.A., 2007, An empirical approach to understanding privacy valuation, HBS Marketing Research Paper 07-075, Available at: $\underline{\text { https://ssrn.c }}$ om/abstract $=982593$

Westin, A.F., 2003. Social and political dimensions of privacy. J. Soc. Issues 59, 431-453.

\section{Footnotes}

Text Footnotes

[1] Consumers' searches and purchases at Internet stores provide firms with a wealth of personal information on gender, age, location, preferences, and other relevant data to identify consumers' types.

[2] Section 2 discusses personal data protection policies both in the EU and in the US

[3] Wathieu and Friedman (2007) present evidence from a large-scale experiment that consumers have a sophisticated understanding of indirect privacy effects, so that they are sensitive to personal data use, especially for fear of price discrimination. Interestingly, some empirical investigations have nonetheless found that more (perceived) control over privacy may lead to more personal data sharing (Brandimarte et al., 2013; Tucker, 2014).

[4] Think of individuals who avoid accepting cookies, registering on websites, using social networks or geolocation devices. 
This terminology reflects Westin's segmentation of the public on consumer privacy issues (see e.g. Westin, 2003). Recently, Lin (2020) has provided empirical evidence suggesting that some consumers have intrinsic privacy preferences (a 'taste for privacy') in addition to instrumental privacy valuations (which can be directly linked to price discrimination).

[6] Similarly, airline and railway companies provide registered users with ancillary services and/or promotional offers. Stores in shopping malls may access personal data after visitors register for free Wi-Fi. These data allow sellers to send targeted offers via SMS-like messages, which may increase purchasing efficiency and satisfaction (Ghose et al., 2020). Users of sport equipment, such as connected athletic gear, may enjoy special services in exchange for access to personal data from smartphones. Thus, the UA HOVR running shoes track a wide array of data, from distance and pace to cadence and stride length. Using this data, the application MapMyRun provides a runner with a personalized coaching experience.

[7] As a benchmark, we consider the case where there is no privacy regulation, and thereby the firm is completely informed about the WTP for the product of privacy pragmatists (even without their explicit consent).

[8] See, in particular, articles 4(11), 6(1), and 7, and recitals 32, 40, 42, 43, 50, and 71 of the Regulation (EU) 2016/679 of the European Parliament and of the Council that is entered into force in May 2018, thereby repealing Directive 95/46/EC

[9] From Recital 32, this could include ticking a box when visiting an Internet website, or choosing technical settings for information society services (silence, pre-ticked boxes, or inactivity should not constitute consent).

[10] The GDPR also strengthens some fundamental rights of individuals, such as the right to be informed, to erasure (be forgotten), and to restrict automated decisions and profiling (see e.g. https://eugdprompliant.com/eu-citizens-rights).

[11] See e.g. The Economist (https://www.economist.com/news/leaders/21739961-gdprs-premise-consumers-should-be-charge-their-own-personal-data-right), America should borrow from Europe's data-privacy law, April 5, 2018.

[12] In contrast, Liu and Shuai (2016) obtain that consumer surplus is lower with duopoly price discrimination.

[13] Users are often offered a zero price for online services, since they pay with their data and attention.

[14] Bo and Manduchi (2017) analyze pros and cons of consumer data provision when it increases competition in the product market. In a different context, Esteban and Hernández (2017) show that a regulatory policy aimed at protecting consumer privacy, by banning the use of direct advertising without permission, may result in lower social welfare.

[15] A number of empirical studies have found that privacy regulation may increase the costs and/or reduce the benefits of data-driven innovation, thereby dampening the incentives to invest (see Goldfarb and Tucker, 2012).

[16] Relatedly, Dosis and Sand-Zantman (2020) study the effects of property rights over the use of data on market outcomes.

[17] Some related studies assess the impact of (third-degree) price discrimination on product quality (see e.g. Alexandrov and Deb, 2012; Nguyen, 2014). They all find that price discrimination improves quality. Notable exceptions are Matteucci and Reverberi $(2017,2018)$, who show that quality may be higher under uniform pricing[Instruction: Please insert here the following statement in parentheses:

(see also D'Annunzio and Reverberi, 2016)]. We add to this literature by considering that privacy regulation affects the amount of information that can be used to set tailored prices.

[18] Consumers are heterogeneous in their basic WTP for the product. However, all consumers sharing data enjoy the same information benefit. Indeed, professionals have a higher WTP for the intrinsic quality of electronic devices and software programs than students have, since the former look for higher performance [Instruction: the word "microchips" NOT in italics]microchips and more powerful calculus functions. On the other hand, all users potentially benefit from ancillary services such as accessible documentation and technical support. This assumption has also been made in contexts where 'quality' depends on the number of users joining a network (Katz and Shapiro, 1985; Crémer et al., 2000), or on downstream firms' ability to use network investments (Foros, 2004; Avenali et al., 2014). More generally, consumers may even be heterogeneous in their valuation of the information benefit. If the marginal consumer values the information benefit less than the average consumer does then, ceteris paribus, the monopolist will probably have less incentives to invest in quality than in the baseline model.

[19] It is usually assumed in the literature that the utility is strictly concave (i.e. $k<1)$ or linear $(k=1)$ in quality, but this is often made for analytical convenience, rather than empirical relevance. Convexity of the utility function (i.e. $k>1$ ) is suitable for some cases, such as ERP systems, where the benefit of add-on services may increase more than proportionally with the sophistication of the core product. Some other papers have considered this possibility, such as Buehler et al. $(2004,2006)$, who study the impact of access regulation and vertical industry structure on infrastructure quality, and Matteucci and Reverberi (2018), who compare the effects of price discrimination and uniform pricing on product quality.

[20] In Section 6.1, we consider the case where the two groups of consumers are of different sizes.

[21] Alternatively, we could consider a model where consumers are heterogeneous across two dimensions, that is, the values of parameter $\theta \in[0,1]$, which is related to the WTP for the intrinsic quality of the product (dimension 1), and of parameter $\beta \in\left\{\beta_{L}, \beta_{H}\right\}$, measuring the nuisance from sharing personal data (dimension 2). Then, the gross utility of consumer $(\theta, \beta)$ would be $U(\theta, \beta)=\theta q+\gamma q^{k} x-\beta x$. If we assume that: (i) consumers are uniformly distributed across dimension 1 , and split evenly across dimension 2; (ii) $\beta_{L}$ is sufficiently small (i.e. tends to zero); and (iii) $\beta_{H}$ is large enough to satisfy $\beta_{H}>\left(\theta q+\gamma q^{k}\right)$; then, the main results of this alternative model specification are similar to the baseline model.

[22] A more general cost function is $C(q)=\phi q^{2}$, with $\phi>0$. We can show that the main results do not depend on the degree of convexity of the cost function, as tuned by the value of $\phi$. Results are available from the authors on request.

[23] Despite the advances in information technology and the widespread availability of personal data, firms are more likely to be able to engage in group pricing for small targeted groups (i.e. personalized pricing) than in perfect price discrimination (Bourreau and de Streel, 2018). In Section 5.3, we discuss the case where $\alpha=1$ (see footnote 32).

[24] Shaw and Vulkan (2012) argue that buyers typically have to register, so sellers immediately know their identity and can access any data they have on them. Ulph and Vulkan (2007) claim that a data agency such as Experian provides a software package enabling websites to recognize customers instantly and sends customers' profiles to retailers. Moreover, the cost of personalizing prices in real time on the Internet is negligible once the appropriate software is installed.

[25] Ezrachi and Stucke (2016) argue that personalized pricing can be achieved through 'personal digital butlers', such as Apple's Siri or Amazon's Alexa. The more buyers rely on butlers, the less likely they are aware of price discrimination.

[26] Montes et al. (2019) provide a list of further examples of documented price discrimination on the Internet, as well as a list of relevant theoretical models where arbitrage is assumed not to be possible.

[27] In many relevant examples, consumers first observe, but do not actually pay, the uniform price posted on the website, and then decide whether to share personal data (e.g. by registering on the website) to be offered tailored prices. In Section 6.2, we consider an alternative timing where the firm sets prices after observing consumers' privacy choices. 
In a corner solution, even the highest-WTP type in group 1 shares data. This occurs when $P(q) \geq \alpha q-(1-\alpha) \gamma q^{k}$. In such a case, $P(q)$ maximizes profit from sales to group 2. Since profit is concave in $P$ then, if the optimal solution of the unconstrained problem does not fulfil the constraint, the optimal price is $P(q)=\alpha q-(1-\alpha) \gamma q^{k}$. Hereafter, we focus on this case, because otherwise the opt-in regime would not affect firm's choices (for formal details, see Section 5).

[29] Both in an interior and a corner solution, $\widetilde{\theta}>0$ is always satisfied, since $P=0$ is never optimal.

[30] Results are available from the authors on request.

[31] A graphical inspection shows that the main results hold for $\bar{k} \leq k \leq 2$, where $\bar{k}>1$, meaning that the information benefit has to be sufficiently convex in quality.

[32] Figs. 1 and 2 also display the results for the case where $\alpha=1$ (that we have excluded in the baseline model). If the firm can perfectly price discriminate, then consumers do not perceive any information benefit, since the firm reaps it all. Thus, consumers share data as long as they would have a negative surplus from remaining anonymous and paying the uniform price. As shown in the figures, when $\alpha=1$ product quality and consumer surplus are lower under the opt-in regime than in the benchmark case (except for $\gamma=1 / 2$, when they are the same). Note that, in a corner solution, perfect price discrimination gives rise to a degenerate case where the uniform price is such that no consumer in group 2 is active.

[33] We have also checked an alternative model version where the total mass of consumers remains unchanged, while the proportion between the two groups is allowed to change. We have found similar results (that are available on request).

[34] Indeed, we find that $n$ has to be very high $(n>35)$ to cancel out any chance of an increase in total consumer surplus.

[35] For a formal analysis, see the Online Appendix.

[36] We find that the critical value $\gamma_{U}(\alpha)$ is also increasing in $\alpha$. Both $\gamma_{L}(\alpha)$ and $\gamma_{U}(\alpha)$ tend to $1 / 2$ as $\alpha$ tends to 1 .

[37] We assume that $\alpha$ is the same for both firms. Consistent with the monopoly model, we assume that $\alpha \geq 1 / 2$.

[38] Indeed, the higher the ability to extract surplus from consumers who share data, the higher firm $H$ 's incentive to increase the uniform price (see ( $B 5$ ) and ( B7) in Appendix B), and the lower the increase in surplus for consumers in group 1.

\section{Highlights}

- We study how the opt-in privacy regime affects product quality and consumer surplus.

- Consumers sharing data benefit from the complementarity between information and quality.

- If the complementarity is strong enough, quality is higher with than without the opt-in regime.

- An increase in product quality is necessary to improve total consumer surplus.

- These results hold both under monopoly and imperfect product market competition.

\section{Appendix Supplementary materials}

Multimedia Component 1

alt-text: Image, application 1

\section{Queries and Answers}

Query: Please confirm that givennames and surnames have been identified correctly

Answer: Yes 